\title{
Synaptic Interactions between Primary Afferent Terminals and GABA and Nitric Oxide-Synthesizing Neurons in Superficial Laminae of the Rat Spinal Cord
}

\author{
Patrizia S. Bernardi, ${ }^{1, a}$ Juli G. Valtschanoff, ${ }^{1}$ Richard J. Weinberg, ${ }^{1}$ Harald H. H. W. Schmidt, ${ }^{2}$ and Aldo \\ Rustioni ${ }^{1}$ \\ 'Department of Cell Biology and Anatomy, University of North Carolina, Chapel Hill, North Carolina 27599 and \\ ${ }^{2}$ Medizinische Universitätsklinik, Klinische Biochemie und Pathobiochemie, W-8700 Würzburg, Germany
}

The superficial laminae (I and II) of the spinal dorsal horn receive small caliber primary afferent fibers responsive to noxious stimulation, and contain local circuit neurons that modulate afferent input. Many of these neurons are GABAergic; about a third of these also synthesize nitric oxide. We identified three main morphological types of primary afferent terminals in superficial laminae after injections of a tracer selective for small caliber afferents into the sciatic nerve of rats. The relative densities of the three types varied through the dorsoventral extent of laminae $I$ and II. Synaptic contacts of each type with GABA- and nitric oxide synthase (NOS)-containing dendrites and axon terminals were determined by preembedding and postembedding immunocytochemistry.

Nonglomerular primary afferent terminals, likely to originate from peptidergic unmyelinated fibers, were not seen in synaptic contact with either GABA- or NOS-containing neurons. Primary afferent terminais at the center of type 1 glomeruli (C1) and at the center of type 2 glomeruli (C2) are likely to originate from unmyelinated and small myelinated fibers, respectively. GABAergic terminals contacted more $C_{2}$ than $C_{1}$ terminals, suggesting more effective presynaptic inhibition of $\mathrm{C}_{2}$ terminals. Many GABAergic terminals were also positive for NOS, but all GABAergic terminals presynaptic to primary afferent terminals were negative for NOS. Only C2 terminals established frequent synapses with NOSpositive dendrites. These results provide morphological evidence for selective inhibitory gating of input to superficial dorsal horn, and suggest that the link between noxious input and nitric oxide-synthesizing neurons, likely to be involved in nociception, may be provided both by direct synaptic contacts of small myelinated fibers onto dorsally extending NOSpositive dendrites, and by unmyelinated fibers via an oligosynaptic pathway.

\footnotetext{
Received Dec. 1, 1993; revised Aug. 5, 1994; accepted Aug. 10, 1994.

We thank K. D. Phend for her expert histological assistance. This work was supported by NINDS Grant 12440 to A.R. and by a fellowship from the Italian Ministry for the University and Scientific Research (Murst 40\%) to P.S.B.

Correspondence should be addressed to Aldo Rustioni, Department of Cell Biology and Anatomy, University of North Carolina, CB\# 7090, Chapel Hill, NC 27599.

Present address: Sezione Istologia ed Anatomia Umana, Dipartimento di Fisiologia e Biochimica Generali, Universitá degli Studi di Milano, 20133 Milano, Italy.

Copyright (C) 1995 Society for Neuroscience $0270-6474 / 95 / 151363-09 \$ 05.00 / 0$
}

[Key words: somatosensation, substantia gelatinosa, presynaptic inhibition, synaptic glomeruli, modulation of nociception]

An extensive literature deals with the morphological and functional properties of primary afferent fibers and their spinal terminations. Special attention has been paid to those afferents terminating in superficial laminae of the dorsal horn because of their involvement in the mediation of pain (Light, 1992). Primary afferents terminating in the superficial dorsal horn that mediate nociceptive input are likely to release glutamate as neurotransmitter (Schneider and Perl, 1985; Schouenborg and Sjölund, 1986; DeBiasi and Rustioni, 1988; Skilling et al., 1988; Yoshimura and Jessell, 1990). These fibers may also release other mediators, including aspartate and neuropeptides (Tracey et al., 1991; Kangrga and Randic, 1991; Light, 1992; Sorkin and McAdoo, 1993).

By combining tracing methods with electron microscopic postembedding immunocylochemistry, we have recently demonstrated enrichment of glutamate and aspartate in different types of primary afferent terminals (PAT) in the superficial laminae of the dorsal horn (Valtschanoff et al., 1994). These include the central (C) terminals of type 1 and type 2 glomeruli, referred to as $\mathrm{C} 1$ and C2, respectively (Ribeiro-da-Silva and Coimbra, 1982; Ribeiro-da-Silva, 1994). Both types predominate in the inner part of lamina II; $\mathrm{Cl}$ terminals may contain dense core vesicles, seldom found in $\mathrm{C} 2$. $\mathrm{C} 1$ terminals stain more densely for glutamate and aspartate than $\mathrm{C} 2$ or a third type, also identified as originating from primary afferents in previous literature (Knyihár-Csillik et al., 1982a; Valtschanoff et al., 1992a; 1994). This type of terminal, which is not involved in glomerular arrangements, predominates in laminae I and outer II and displays numerous dense core vesicles.

We determine here the relations of PAT to GABAergic profiles in superficial laminae in order to explore mechanisms of central modulation of these afferents. Recent work has demonstrated the presence of neurons in the superficial laminae of the dorsal horn that may synthesize nitric oxide (NO, Valtschanoff et al., 1992b; Dun et al., 1993); most such neurons are GABAergic (Valtschanoff et al., 1992c; Spike et al., 1993). The involvement of NO in dorsal horn mechanisms responsible for nociception and hyperalgesia seems likely (Meller and Gebhart, 1993). Therefore, in this study we also investigate the relations of the different types of PAT in the superficial dorsal horn with neurons containing NO synthase (NOS). 


\section{Materials and Methods}

Observations were from seven male Sprague-Dawley rats (Charles River, $150-500 \mathrm{gm})$ anesthetized with sodium pentobarbital $(40 \mathrm{mg} / \mathrm{kg})$. The left sciatic nerve was injected above the knee with $2 \%$ WGA-HRP (Sigma) dissolved in 5\% dimethyl sulfoxide through a $30 \mathrm{G}$ needle attached with thin polyethylene tubing to a Hamilton microsyringe. After $2-3 \mathrm{~d}$, rats were reanesthetized and perfused intra-aortically with 100 $\mathrm{ml}$ of heparinized saline, followed by $500 \mathrm{ml}$ of a freshly prepared mixture of $2.5 \%$ glutaraldehyde, $0.5 \%$ paraformaldehyde and $0.1 \%$ picric acid in $0.1 \mathrm{M}$ phosphate buffer, $\mathrm{pH} 7.3$ (PB). Lumbar segments associated with the sciatic nerve were removed, postfixed for $2 \mathrm{hr}$ in the same fixative, and stored in PB. Fifty micrometer thick transverse sections were cut on a Vibratome and reacted according to a tetramethyl benzidine/tungstate (TMB/T) protocol to reveal peroxidase (Weinberg and Van Eyck, 1991). Sections from three rats with dense anterograde labeling (usually from L4) were further processed for either postembedding immunostaining for GABA, or preembedding immunostaining for NOS, or a combination of the two. To avoid confusion in the text, the term "stained" refers to immunopositive elements, whether revealed by diaminobenzidine (DAB, in material processed for preembedding immunocytochemistry), or by gold particles (in postembedding immunocytochemistry), and the term "labeled" refers to the presence of the anterograde tracer.

For postembedding staining for GABA sections were immersed in $1 \% \mathrm{OsO}_{4}$ for $1 \mathrm{hr}$, stained in $1 \%$ uranyl acetate for $1 \mathrm{hr}$ and wafer embedded in Epon-Spurr. For light microscopy, $1 \mu \mathrm{m}$ thick sections were mounted on gelatin-coated slides and stained for GABA, and then were etched in potassium methoxide/propylene oxide for $2 \mathrm{~min}$, pretreated in $0.3 \%$ hydrogen peroxide, incubated overnight in rabbit antiGABA antibody (Sigma, 1:100 in phosphate-buffered saline, pH 7.5), then for $1 \mathrm{hr}$ in biotinylated anti-rabbit antibody (Vector, 1:200) and for $1 \mathrm{hr}$ in ExtrAvidin-peroxidase (Sigma 1:5000). For electron microscopy, small pieces of tissue were excised from the central portion of laminae I-III of L4 where the bulk of sciatic afferents to superficial laminae terminate (LaMotte et al., 1991) and glued to a plastic block for ultramicrotomy. Thin sections were mounted on uncoated nickel mesh grids and processed as described by Phend et al. (1992), as follows. Grids were washed with Tris-buffered saline containing $0.1 \%$ Triton $\mathrm{X}-100(\mathrm{TBS}+\mathrm{T}), \mathrm{pH} 7.6$, incubated overnight in the primary antibody $(1: 10,000)$, rinsed in TBS $+\mathrm{T}$, $\mathrm{pH} 7.6$, transferred in TBS $+\mathrm{T}, \mathrm{pH} \mathrm{8.2,}$ incubated in secondary antibody for $1 \mathrm{hr}$, rinsed, and dried. Goat antirabbit IgG conjugated to $20 \mathrm{~nm}$ or $30 \mathrm{~nm}$ gold particles (E-Y Labs, 1:25 in TBS+T, pH 8.2) was used as secondary antibody. Grids were counterstained with $5 \%$ uranyl acetate and Sato's lead and examined on a JEOL 200CX electron microscope. Preabsorption of the anti-GABA serum with free GABA blocked the postembedding staining.

For preembedding staining for NOS, sections pretreated in $1 \%$ sodium borohydride for $30 \mathrm{~min}$ were permeabilized with $50 \%$ ethanol for 10 min, incubated in $3 \%$ hydrogen peroxide (to inactivate endogenous peroxidases), and then in $10 \%$ normal goat serum (to block secondary antibody binding sites), and transferred into anti-NOS antibody (\#6761$8,1: 5000)$ for overnight incubation at room temperature. Sections were then incubated $1 \mathrm{hr}$ in biotinylated anti-rabbit antibody (Vector, 1:200) and $1 \mathrm{hr}$ in ExtrAvidin-peroxidase complex (1:5000). Binding sites were visualized with $\mathrm{DAB}$. The antibody for NOS was characterized in Schmidt et al. (1992). In the present material we verified that (1) NOS immunoreactivity coexisted, at light microscopic level, with histochemistry for NADPH diaphorase, a widely-accepted marker for NOS, and (2) NOS immunoreactivity gradually disappeared at progressively higher dilutions of the primary antiserum.

Camera lucida drawings from $1 \mu \mathrm{m}$ thick sections stained with thionin supplied reference landmarks used to identify laminar boundaries in thin sections. Quantitative data were collected on the screen of the electron microscope $(12,000 \times)$ or from prints enlarged from $20,000 \times$ negatives. Details on the samples employed for quantitative data are given with the description of the results.

\section{Results}

\section{Morphology and distribution of labeled PAT}

At the light microscope, the anterograde labeling was most prominent in lamina II, as expected from previous observations. This lamina consists of an outer cell-dense lamina IIo, and an inner cell-poor lamina IIi. In the cervical cord of rats, Ribeiro-
da-Silva and Coimbra (1982) subdivided lamina II into three sublaminae: IIA $(20 \mu \mathrm{m})$, IIBd (dorsal $20 \mu \mathrm{m})$, and IIBv (ventral, $45 \mu \mathrm{m}$ ). In the lumbar cord, lamina IIo corresponds to lamina IIA, and lamina IIi to lamina IIBd and IIBv. Labeling in our material was mainly in a band of the spinal gray identified, by the absence of myelinated fibers and the sparse cellular density, as IIi; labeling was densest in its dorsal portion. Laminae I and IIo contained sparse labeling, and the gray matter ventral to lamina II appeared virtually free of anterograde tracer (Fig. $1 A$ ).

At the electron microscope, labeled axons and their terminals were identified by the presence of amorphous deposits or rectangular crystals of TMB/T reaction product. Only terminals in which the reaction product did not obscurc the main cytological features were included for quantitative analysis. We identified three morphological kinds of PAT: (1) sinuous terminals (CI) with dark axoplasm, sparse mitochondria and vesicles of variable size (Fig. $1 B$ ), sometimes including dense core vesicles (Fig. $1 C$ ), forming the central element of synaptic glomeruli of type 1 (Ribeiro-da-Silva and Coimbra, 1982); (2) scalloped terminals (C2) with light axoplasm, loosely packed uniform clear vesicles and several mitochondria, in some cases containing neurofilaments (Fig. $1 D, E$ ), and forming the central element of synaptic glomeruli of type 2 (Ribeiro-da-Silva and Coimbra, 1982); (3) terminals, mostly dome-shaped, with light axoplasm containing both clear and dense core vesicles, not involved in glomerular arrangements (Fig. $1 F$ ).

That the three kinds of terminals are not uniformly distributed in the superficial lamina has been reported in material without tracer labeling (Ribeiro-da-Silva, 1994). Out of 178 tallied labeled terminals, the majority were C1s (mainly in IIi); about $10 \%$ were $\mathrm{C} 2 \mathrm{~s}$ (exclusively in IIi), and about $25 \%$ were nonglomerular PAT (in I and IIo). Accurate estimates of terminal distribution are complicated by stereological considerations: smaller boutons, especially nonglomerular ones, are likely to be underrepresented. Also, not all terminals with morphological features of PAT were labeled in the plane of the section. This is in part to be expected as terminals of saphenous nerve are intermingled with those from the sciatic nerve in the central portions of the superficial laminae of L4 (LaMotte et al., 1991). However, in several sections from a rat in which $\mathrm{Cl}$ and $\mathrm{C} 2$ terminals were identified by their characteristic morphology, about $70 \%$ of $\mathrm{C} 1$ and only about $15 \%$ of $\mathrm{C} 2$ terminals were labeled. The percentage of nonglomerular PAT labeled was not determined, as unlabeled terminals of this type are likely to include those from intrinsic peptidergic neurons (Ribeiro-daSilva et al., 1991).

\section{GABA-positive profiles}

Light microscopic postembedding immunocytochemistry for GABA confirmed previous observations demonstrating GABAergic neurons and terminals in lamina II of the dorsal horn (Fig. 2A; Barber et al., 1978; Todd and Spike, 1993). At the electron microscope, gold particles coding for GABA were enriched over some perikarya, dendrites, and axon terminals. In terminals they were dense over mitochondria and clear synaptic vesicles, though not all vesicles were overlaid by particles. GABAstained terminals were small and dome-shaped with many flat or round clear synaptic vesicles, few mitochondria, and occasional dense core vesicles (Fig. $2 B-F$ ). They made single synaptic contacts of the symmetric type onto perikarya, dendrites, and axon terminals. Many GABA-positive dendrites contained vesicles. Vesicle-containing dendrites were small; their vesicles 

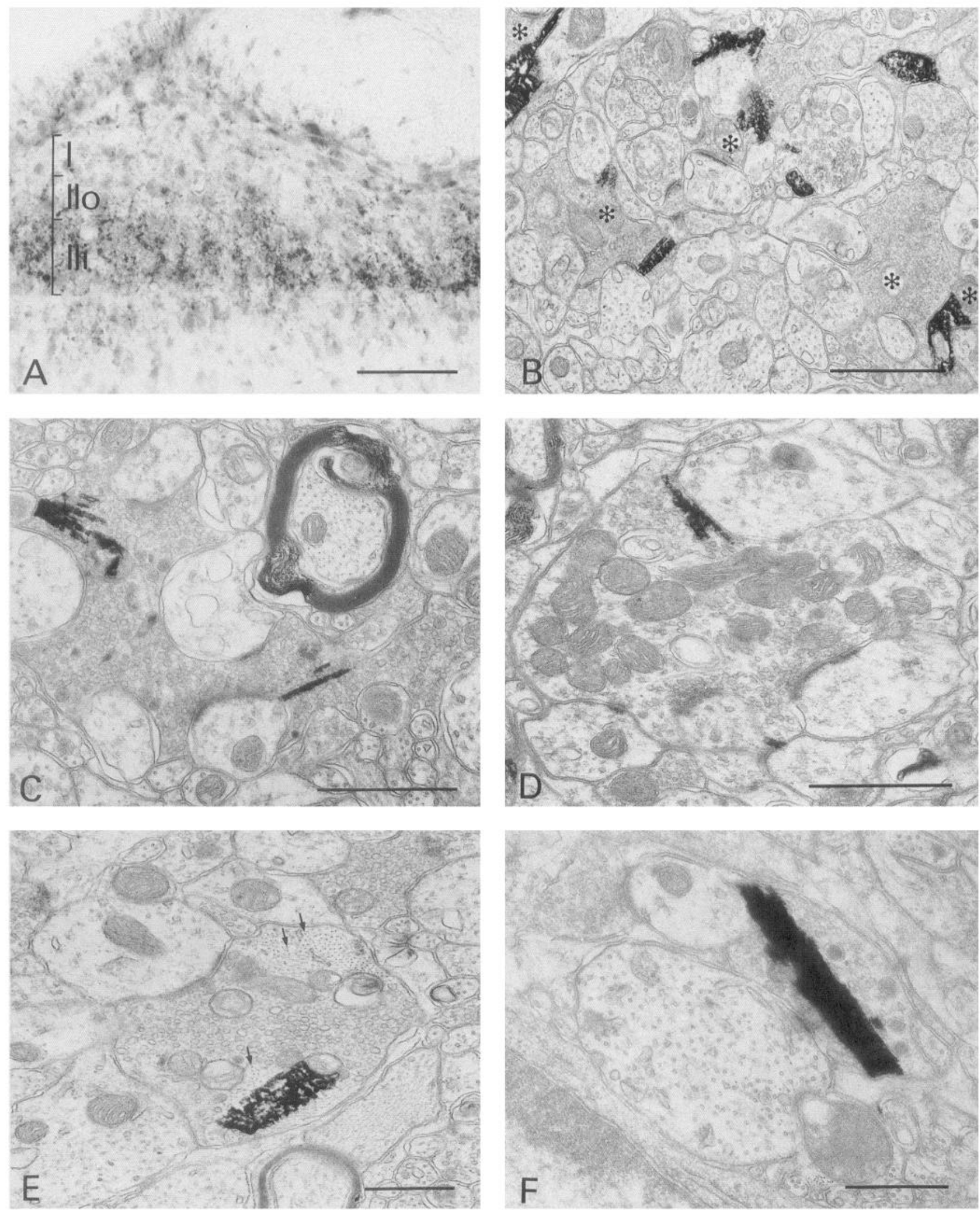

Figure 1. Anterograde labeling in superficial laminae of the dorsal horn after sciatic injection of WGA-HRP. A, Low-power photomicrograph shows that labeling is concentrated in the inner part of lamina II (IIi) at L4. Laminar boundaries as defined in the text. B-E, Electron microscopy of different types of labeled primary afferent terminals. B, Dark sinuous (C1) terminals (asterisks) in lamina II at L4, four labeled and one unlabeled. Terminals lacking dense core vesicles are the most frequently labeled among $C 1 \mathrm{~s}$. $C$, Labeled $\mathrm{Cl}$ terminal containing dense core vesicles. $D$, Labeled light scalloped (C2) terminal, containing many mitochondria, at the center of a type 2 glomerulus; this $\mathrm{C} 2$ terminal does not display neurofilaments in the plane of the section. $E$, Labeled $\mathrm{C} 2$ terminal containing neurofilaments (arrows). $F$, Small nonglomerular terminal in lamina IIo containing many dense core vesicles. Scale bars: $A, 50 \mu \mathrm{m} ; B-D, 1 \mu \mathrm{m} ; D$ and $F, 0.5 \mu \mathrm{m}$. 

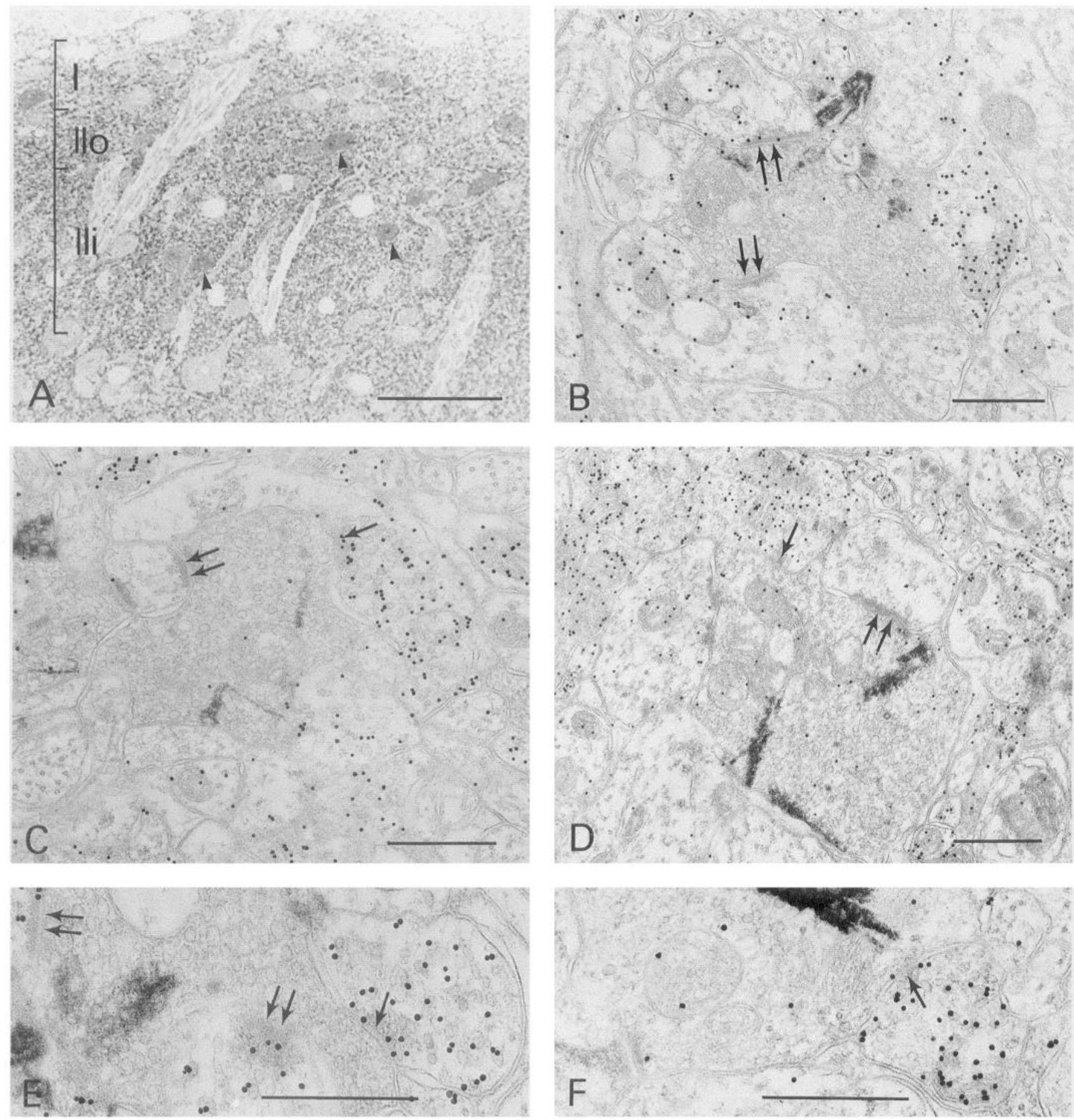

Figure 2. Immunostaining for GABA in superficial laminae. In $A$ postembedding light microscopic staining was performed on $1 \mu \mathrm{m}$ thick plastic section; examples of stained perikarya are indicated by arrowheads. In $B-F$, electron microscopic postembedding staining for GABA is combined with anterograde labeling of primary afferent terminals. Double arrows indicate synapses made by primary afferent terminals upon postsynaptic profiles. Single arrows indicate synapses made by GABA-positive endings upon primary afferent terminals. $B, \mathrm{~A} \mathrm{Cl}$ terminal without dense core vesicles is presynaptic to two dendrites enriched in gold particles. $C$ and $D$ show $\mathrm{Cl}(C)$ and $\mathrm{C} 2(D)$ primary afferent terminals postsynaptic to GABA-positive endings. $E$ and $F$, Higher-power photomicrographs to show synapses of GABA-positive endings upon $C 1(E)$ and $C 2(F)$ terminals. Scale bars: $A, 50 \mu \mathrm{m} ; B-F, 0.5 \mu \mathrm{m}$.

were relatively few and clustered at synaptic sites, rather than numerous and widespread, as in axon terminals (Fig. 2D).

Density of gold particles in GABA-positive profiles was generally highest over axon terminals, and higher over vesiclecontaining dendrites than other dendrites. Vesicle-containing profiles that were enriched in GABA could be identified by their higher density of gold particles compared to other profiles (see Materials and Methods; Phend et al., 1992; Valtschanoff et al., 1994). For quantitative analysis, immunopositivity for GABA was defined as density of gold particles at least three times higher 

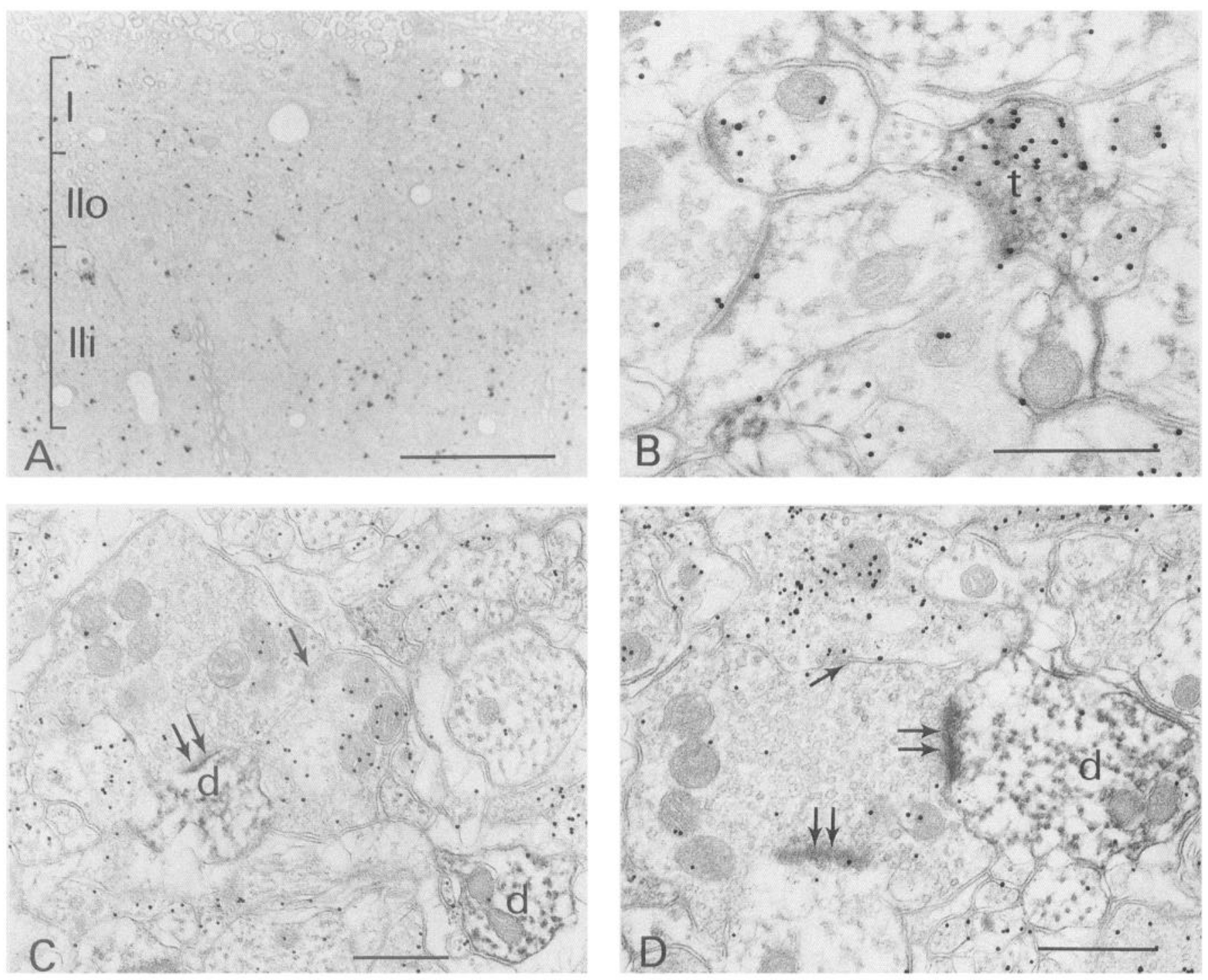

Figure 3. Immunostaining for NOS in superficial laminae. In $A$, light photomicrograph of a $1 \mu \mathrm{m}$ thick plastic section from a $50 \mu \mathrm{m}$ thick section processed for preembedding staining for NOS; staining consists mainly of dark dots corresponding to dendrites, axons, and terminals; arrows as in Figure 2. In $B-D$, Staining for NOS is combined with postembedding staining for GABA. $B$, An NOS-positive terminal $(t)$ is also GABA-positive and contacts a negative dendrite. $C, \mathrm{~A} \mathrm{C} 2$ terminal contacts an NOS-positive dendrite $(d)$; the terminal receives a synaptic contact from a GABApositive ending. $D$, Another $\mathrm{C} 2$ terminal contacts an NOS-positive dendrite $(d)$; a GABA-positive ending is presynaptic to the $\mathrm{C} 2$ terminal. Scale bars: $A, 50 \mu \mathrm{m} ; B-D, 0.5 \mu \mathrm{m}$.

than in adjacent profiles (Valtschanoff et al., 1994). None of the PAT, whether identified by anterograde labeling or by morphological characteristics, were stained for GABA.

GABA-positive profiles were often in contact with PAT. The relation of GABA-positive profiles to PAT differed according to the type, as shown by the quantitative data of Table 1. C1 terminals were more frequently presynaptic to GABA-positive profiles than $\mathrm{C} 2$ (Fig. $2 C-F$ ). Of $15 \mathrm{Cl}$ terminals presynaptic to GABA-positive profiles in this data set, 3 were presynaptic to conventional dendrites (Fig. $2 C$ ), 11 to vesicle-containing dendrites (Fig. $2 B$ ) and 1 contacted one of each kind. Of the seven $\mathrm{C} 2$ terminals presynaptic to GABA-positive profiles, five were presynaptic to conventional dendrites, and two to vesiclecontaining dendrites. Some of the GABA-positive profiles postsynaptic to PAT were also presynaptic to unstained dendrites (Fig. 2D). C1 terminals were much less likely than $\mathrm{C} 2$ to be postsynaptic to GABA-positive profiles, generally axon termi- nals (Fig. 2C,E). In only one case was a $\mathrm{Cl}$ postsynaptic to a GABA-positive profile recognizable as a vesicle-containing dendrite.

Table 1. PAT and GABA-positive profiles

\begin{tabular}{llll}
$\begin{array}{l}\text { Type of } \\
\text { PAT }\end{array}$ & $n$ & $\begin{array}{l}\text { Presynaptic } \\
\text { to GABA }\end{array}$ & $\begin{array}{l}\text { Postsynaptic } \\
\text { to GABA }\end{array}$ \\
\hline C1 & 54 & $15(28 \%)$ & $3(6 \%)$ \\
C2 & 42 & $7(17 \%)$ & $9(21 \%)$
\end{tabular}

Data are from 96 photomicrographs, taken from superficial laminae in two rats; percentages from the total number of tallied PAT are in parentheses. PAT types are according to morphological criteria given in the text: $\mathrm{Cl}$ included 42 anterogradely labeled and 12 unlabeled terminals, and C2 included 6 labeled and 36 unlabeled. The majority of PAT were in nonsynaptic contact with or did not contact GABA-positive profiles. No contacts between nonglomerular terminals and GABA-positive profiles were seen. 


\section{Presynaptic}

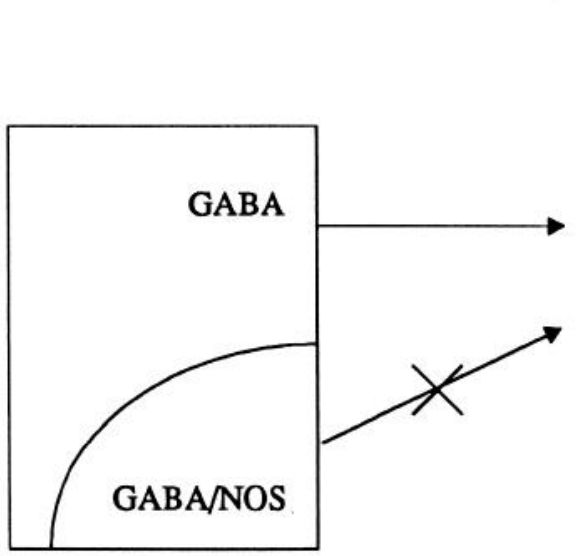

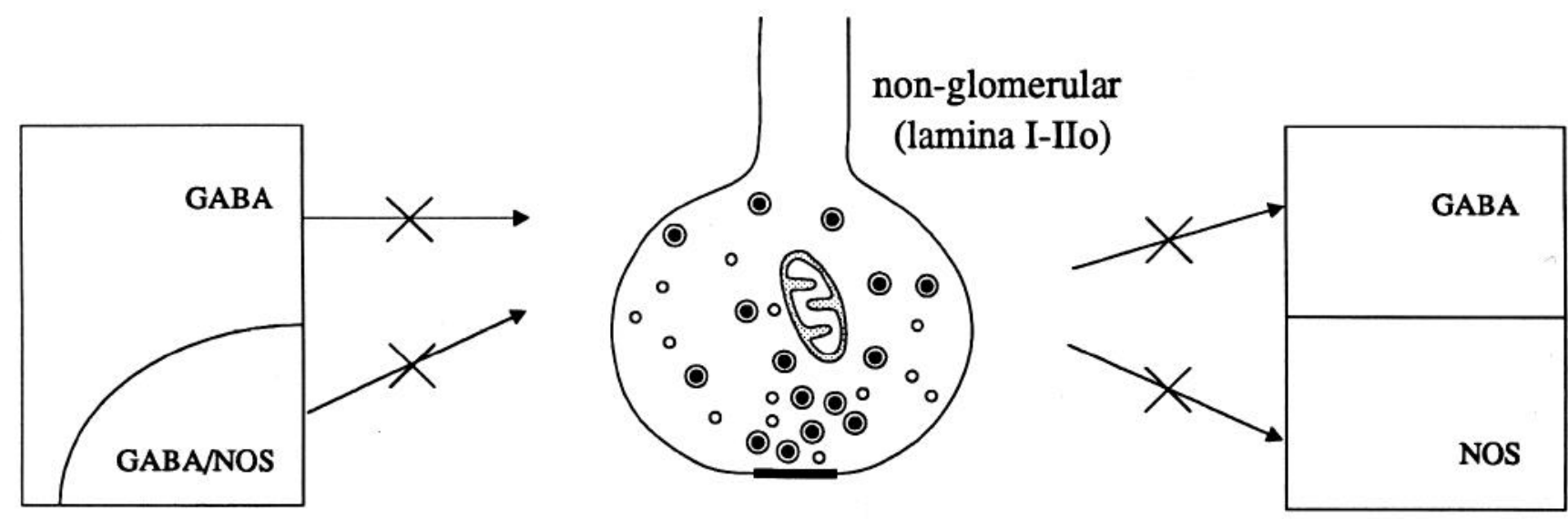

\section{Primary afferents}

Postsynaptic

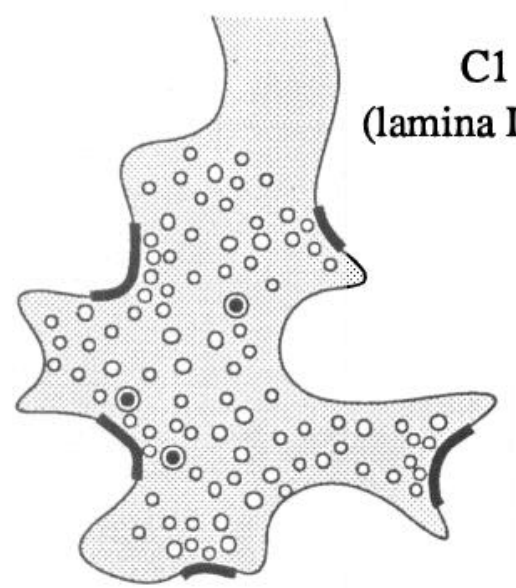

C1
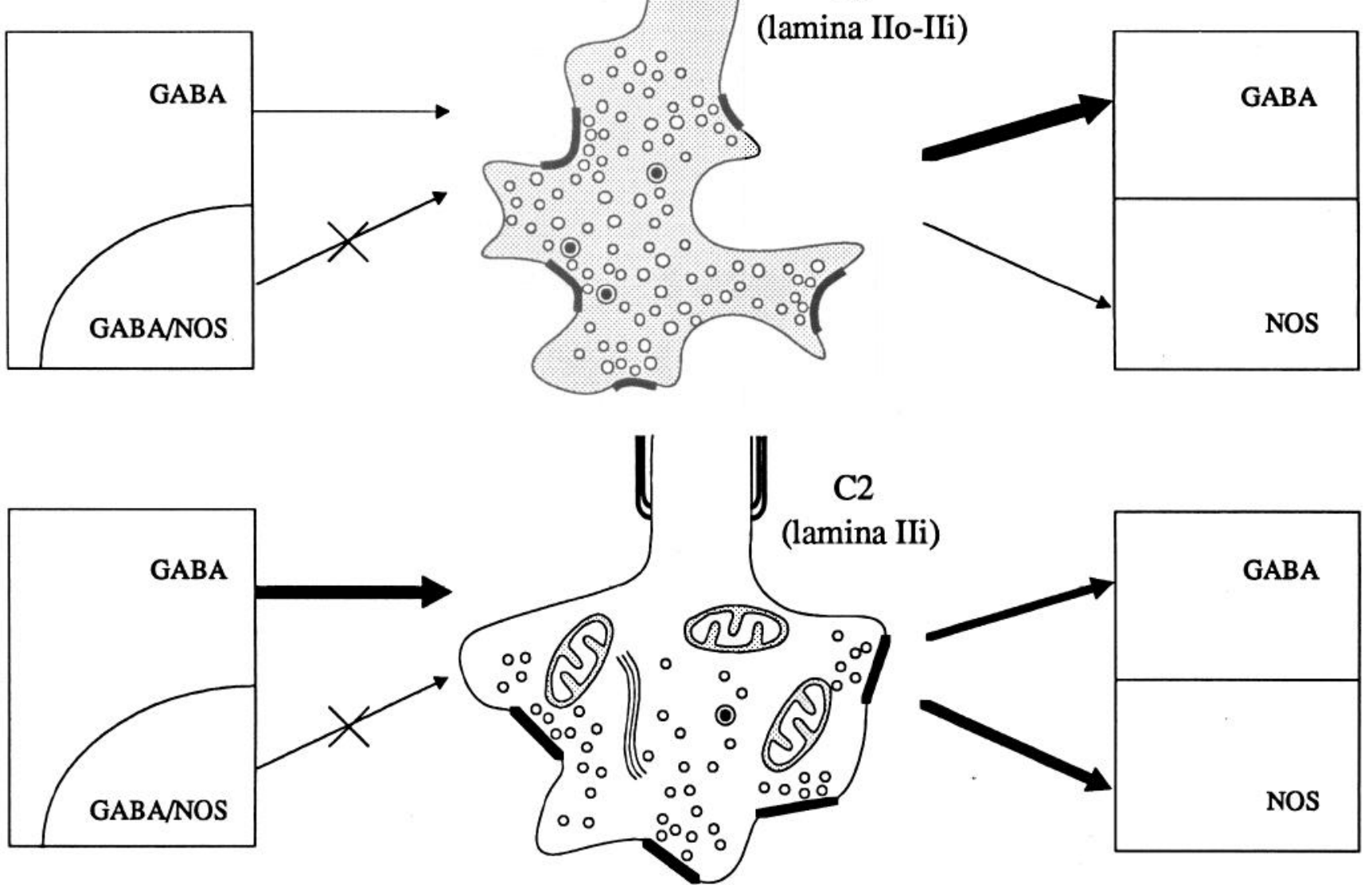

Figure 4. Summary and schematic representation of the results discussed in the text.

\section{NOS-positive profiles}

The laminar distribution of NOS immunoreactivity was as reported previously (Valtschanoff et al., 1992b; Dun et al., 1993; Fig. $3 \mathrm{~A}$ ). At the electron microscope it was present in perikarya, dendrites, myelinated and unmyelinated axons, and synaptic terminals. NOS-positive perikarya were small, with deeply indented nuclei and a narrow ring of cytoplasm. Electron-dense reaction product was associated with membranes; neurofilaments and microtubules of immunopositive axons and dendrites were also stained. Staining was not observed in endothelial cells or astrocytes. NOS-positive terminals were small, with few mitochondria and symmetric active zones. They contained a uniform population of clear vesicles and occasional dense core vesicles (Fig. $3 B$ ). Double staining revealed frequent colocalization of NOS with GABA as previously demonstrated (Fig. 
$3 B$; Valtschanoff et al., 1992c). However, many GABA-positive profiles were NOS-negative, and only 8 of 112 randomly selected NOS-positive dendrites were noticeably enriched in GABA.

None of the PAT exhibited NOS immunopositivity. Axodendritic but not axoaxonic synaptic contacts were seen between PAT and NOS-positive profiles. Contacts between $\mathrm{Cl}$ terminals and NOS-positive dendrites were very sparse and no relations existed between nonglomerular boutons and NOS-positive dendrites. Contacts onto NOS-positive dendrites were commonly seen with $\mathrm{C} 2$ terminals (Fig. $3 C, D$ ). Counts were made from two animals from the region (IIi-dorsal III) where the highest density of NOS-positive profiles was found: out of a total of 35 C2 terminals, 9 (26\%) contacted NOS-positive dendrites.

The relations of three types of PAT with GABAergic and NOS-positive profiles are summarized in Figure 4.

\section{Discussion}

Types of terminals labeled by WGA-HRP

For identification of PAT we used the transport of WGA-HRP injected in the sciatic nerve. Our observations were therefore limited to terminals in the superficial laminae of the lumbar enlargement, presumably endings of unmyelinated and small myelinated primary afferent fibers. A selectivity of WGA-HRP for these fibers has been reported previously and related to the affinity of this tracer for $\mathrm{N}$-acetyl-D-glucosamine and neuraminic acid (Robertson and Grant, 1985; Valtschanoff et al., 1992a). While it is generally agreed that WGA-HRP is taken up by unmyelinated fibers in the peripheral nerve, small myelinated fibers may also take up the tracer to a variable extent (depending on concentration, survival time, solvent, etc.). We observed labeling only in unmyelinated fibers in transverse sections of L4 dorsal root 2-3 d after injection of the sciatic nerve (Valtschanoff et al., 1992a). Given the predominance of labeled $\mathrm{Cl}$ terminals, likely to be endings of unmyelinated fibers ( $\mathrm{Ri}-$ beiro-da-Silva and Coimbra, 1984; Ribeiro-da-Silva, 1994), the present results confirm selectivity of WGA-HRP for these fibers in the PNS. However, the labeling of some $\mathrm{C} 2$ terminals, perhaps arising from small myelinated primary afferents (Coimbra et al., 1984), supports previous indications that the selectivity may not be absolute (LaMotte et al., 1991). Nonglomerular terminals, predominating in lamina I and IIo and characterized by the presence of dense core vesicles, are likely to include peptidergic endings of unmyelinated primary afferents; it is, therefore, not surprising that they were labeled by WGA-HRP.

The identification of three main classes of PAT is a foundation for further investigation on synaptic mechanisms in superficial laminae. These three classes of terminals have been identified in superficial laminae of the rat cervical cord (Ribeiro-da-Silva and Coimbra, 1982; Ribeiro-da-Silva et al., 1985; Cruz et al., 1993), and resemble those identified in cats and monkeys (Knyihár-Csillik et al., 1982a; Maxwell and Réthelyi, 1987; Carlton and Hayes, 1990; Hayes and Carlton, 1992) though their contacts with adjacent dendrites and axon terminals may be more elaborate in these species than in rats. The relative laminar distribution of the three types reported here is generally consistent with that in the cervical cord of rats (Ribeiro-da-Silva and Coimbra, 1982) and the lumbar cord of monkeys (KnyihárCsillik et al., 1982a). Most PAT in lamina I were nonglomerular; moving ventrally these terminals were gradually replaced by $\mathrm{C} 1 \mathrm{~s} . \mathrm{Cl}$ and $\mathrm{C} 2$ terminals were intermingled in $\mathrm{IIi}$, with $\mathrm{C} 1$ decreasing and $\mathrm{C} 2$ increasing from dorsal to ventral within this sublamina (see also Ribeiro-da-Silva, 1994). Nonglomerular and some $\mathrm{C} 1$ terminals contained dense core in agreement with the high density of immunostaining for peptides in laminac I and IIo of the rat lumbosacral cord (Light, 1992).

\section{Synaptic relations to GABAergic neurons}

The present results show that the three types of terminals have different synaptic relations with GABAergic neurons. $\mathrm{Cl}$ terminals contacted more GABAergic dendrites than did $\mathrm{C} 2$ terminals, whereas $\mathrm{C} 2$ terminals were more likely than $\mathrm{C} 1$ terminals to be postsynaptic to GABAergic processes. Since $\mathrm{Cl}$ terminals contacted a higher fraction of vesicle-containing dendrites, more likely than other dendrites to be identified as GABApositive, it is possible that $\mathrm{C} 1$ and $\mathrm{C} 2$ terminals contact GABAergic dendrites in comparable numbers. Given the large size of these primary afferent terminals, stereological considerations suggest that all terminals of both $\mathrm{C} 1$ and $\mathrm{C} 2$ may contact GABAergic dendrites, and that all $\mathrm{C} 2$ terminals may be postsynaptic to a GABAergic terminal.

These results are consistent with previous morphological studies in rodents and primates showing that $\mathrm{C} 2$ terminals arc more frequently postsynaptic to axon terminals than are $\mathrm{Cl}$ (Knyihár-Csillik et al., 1982b; Ribeiro-da-Silva et al., 1985; Hiura et al., 1991; Cruz et al., 1993). Terminals of different functional classes in the superficial laminae of the spinal cord of monkeys may also differ in their relations with presynaptic GABAergic profiles. Whereas boutons of $\mathrm{A} \delta$ (small myelinated) fibers were consistently postsynaptic to GABAergic axon terminals (Alvarez et al., 1992), none of the boutons of the two intracellularly filled $\mathrm{C}$ fibers examined were postsynaptic to such terminals (Alvarez et al., 1993). Comparison between these results and the present data are complicated by differences in sample collection (limited but functionally identified in monkeys, extensive but without functional identification in the present material) and species differences (e.g., glomeruli in monkeys are more complex than in rats). However, the present data and the studies reviewed above concur in suggesting that peptidergic unmyelinated $(\mathrm{C})$ fibers giving rise to nonglomerular terminals in laminae I and IIo lack GABAergic modulation, whereas $\mathrm{Cl}$ terminals in Ili receive modest GABAergic modulation, and C2 terminals in IIi receive strong GABAergic modulation. This differential inhibitory modulation may be associated with the relatively poor spatiotemporal resolution characteristic of $\mathrm{C}$ fibers (Willis and Coggeshall, 1991). The present results suggest that, at least in rodents, presynaptic inhibition plays a less significant role in the spinal gating of nociception than is the case for other cutaneous modalities Based on recent evidence, GABA mechanisms modulating pain sensibility differ, compared to other cutaneous modalities, also at thalamic level (Ralston and Ralston, 1994).

\section{Synaptic relations to NO-synthesizing neurons}

GABAergic neurons in superficial laminae are not homogeneous. A complex pattern of colocalization of GABA with other putative neurotransmitters is emerging: many GABAergic neurons also contain glycine, $\mathrm{ACh}$, and/or various peptides ( $\mathrm{Ri}-$ beiro-da-Silva and Cuello, 1990; Doyle and Maxwell, 1993; Spike et al., 1993). About one-third of GABAergic neurons in the superficial dorsal horn contain NOS, the enzyme responsible for the synthesis of NO (Valtschanoff et al., 1992c; Spike et al., 1993). These neurons may be involved in the mediation of nociception and hyperalgesia (Meller and Gebhart, 1993). Changes in synaptic efficacy associated with hyperalgesia may 
share features in common with higher centers, as NOS coexists with GABA also in interneurons in hippocampus and cortex, where NO is implicated in long-term potentiation (Valtschanoff et al., 1993a,b).

NOS was not present in GABA-positive terminals presynaptic to PAT; thus GABA-positive neurons involved in presynaptic inhibition are unlikely to synthesize NO. C2 terminals commonly contacted NOS-positive dendrites, in contrast to the other two types. NO may be released from dendrites in a nonsynaptic manner, possibly diffusing a considerable distance to reach its target. Neuronal NOS is a calcium-dependent enzyme with slow kinetics (Nathan, 1992). Therefore, synapses onto NOS-positive dendrites may be able to raise calcium sufficiently to trigger NO release only during sustained intense activity. Since NOS-positive neurons are concentrated in IIi and dorsal lamina III, ventral to the major focus of noxious input (Light, 1992; Valtschanoffet al., 1992c), the link between noxious input and NO may be provided both by direct synaptic contacts of A $\delta$ fibers onto dorsally extending NOS-positive dendrites, and by unmyelinated fibers via oligosynaptic pathways. In addition, peptides released from $\mathrm{Cl}$ and nonglomerular terminals can also increase intracellular calcium levels (Womack et al., 1988). During sustained noxious stimulation peptides may diffuse ventrally (Duggan et al., 1990) and act synergistically to trigger NO synthesis.

\section{References}

Alvarez FJ, Kavookjian AM, Light AR (1992) Synaptic interactions between GABA-immunoreactive profiles and the terminals of functionally defined myelinated nociceptors in the monkey and cat spinal cord. J Neurosci 12:2901-2917.

Alvarez. FJ, Kavookjian AM, Light AR (1993) Ultrastructural morphology, synaptic relationships, and CGRP immunoreactivity of physiologically identified C-fiber terminals in the monkey spinal cord. J Comp Neurol 329:472-490.

Barber RP, Vaughn JE, Saito K, McLaughlin BJ, Roberts E (1978) GABAergic terminals are presynaptic to primary afferent terminals in the substantia gelatinosa of the rat spinal cord. Brain Res 141:3555.

Carlton SM, Hayes ES (1990) Light microscopic and ultrastructural analysis of GABA-immunoreactive profiles in the monkey spinal cord. J Comp Neurol 300:162-182.

Coimbra A, Ribeiro-da-Silva A, Pignatelli D (1984) Effects of dorsal rhizotomy on the several types of primary afferent terminals in laminae I-III of the rat spinal cord. An electron microscope study. Anat Embryol 170:279-287

Cruz F, Lima D, Coimbra A (1993) Periterminal organization of primary afferents in laminae I and IIo of the rat spinal cord, as shown after anterograde HRP-labeling. J Neurocytol 22:191-204.

DeBiasi S, Rustioni A (1988) Glutamate and substance P coexist in primary afferent terminals in the superficial laminae of spinal cord. Proc Natl Acad Sci USA 85:7820-7824.

Doyle CA, Maxwell DJ (1993) Neuropeptide Y-immunoreactive terminals form axo-axonic synaptic arrangements in the substantia gelatinosa (lamina II) of the cat spinal dorsal horn. Brain Kes 603:157161.

Duggan AW, Hope PJ, Jarrott B, Schaible HG, Fleetwood-Walker SM (1990) Release, spread and persistence of immunoreactive neurokinin $\mathrm{A}$ in the dorsal horn of the cat following noxious cutaneous stimulation. Studies with antibody microprobes. Neuroscience 35: 195-202.

Dun NJ, Dun SL, Wu SY, Forstermann U, Schmidt HHHW, Tseng LF (1993) Nitric oxide synthase immunoreactivity in the rat, mouse, cat and squirrel monkey spinal cord. Neuroscience 54:845-857.

Hayes ES, Carlton SM (1992) Primary afferent interactions: analysis of calcitonin gene-related peptide-immunoreactive terminals in contact with unlabeled and GABA-immunoreactive profiles in the monkey dorsal horn. Neuroscience 47:873-896.

Hiura A, Ishizuka H, Villalobos EL (1991) GABAergic neurons in the mouse superficial dorsal horn with special emphasis on their relation to primary afferent central terminals. Arch Histol Cytol 54:195-206.

Kangrga I, Randic M (1991) Outflow of endogenous aspartate and glutamate from the rat spinal dorsal horn in vitro by activation of low- and high-threshold primary afferent fibers. Modulation by $\mu$ opioids. Brain Res 553:347-352.

Knyihár-Csillik E, Csillik B, Rakic P (1982a) Ultrastructure of normal and degenerating glomerular terminals of dorsal root axons in the substantia gelatinosa of the Rhesus monkey. J Comp Neurol 210: 357-375.

Knyihár-Csillik E, Csillik B, Rakic P (1982b) Periterminal synaptology of dorsal root glomerular terminals in the substantia gelatinosa of the spinal cord in Rhesus monkey. J Comp Neurol 210:376-399.

LaMotte CC, Kapadia SE, Shapiro CM (1991) Central projections of the sciatic, saphenous, median and ulnar nerves of the rat demonstrated by transganglionic transport of choleragenoid-HRP (B-HRP) and wheat germ agglutinin-HRP (WGA-HRP). J Comp Neurol 311 : $546-562$.

Light A (1992) The initial processing of pain and its descending control: spinal and trigeminal systems. New York: Karger.

Maxwell DJ, Réthelyi M (1987) Ultrastructure and synaptic connections of cutaneous afferent fibres in the spinal cord. Trends Neurosci 10:117-123.

Meller ST, Gebhart GF (1993) Nitric oxide (NO) and nociceptive processing in the spinal cord. Pain 52:127-136.

Nathan C (1992) Nitric oxide as a secretory product of mammalian cells. FASEB J 6:3051-3064.

Phend KD, Weinberg RJ, Rustioni A (1992) Techniques to optimize post-embedding single and double staining for amino acid neurotransmitters. J Histochem Cytochem 40:1011-1020.

Ralston HJ, Ralston DB (1994) Medial lemniscal and spinal projections to the macaque thalamus: an electron microscopic study of differing GABAergic circuitry serving thalamic somatosensory mechanisms. J Neurosci 14:2485-2502.

Ribeiro-da-Silva A (1994) Substantia gelatinosa of spinal cord. In: The rat nervous system, 2d ed (Paxinos G, ed), in press. New York: Academic.

Ribeiro-da-Silva A, Coimbra A (1982) Two types of synaptic glomeruli and their distribution in laminae I-III of the rat spinal cord. J Comp Neurol 209:176-186.

Ribeiro-da-Silva A, Coimbra A (1984) Capsaicin causes selective damage to type I synaptic glomeruli in rat substantia gelatinosa. Brain Res 290:380-383.

Ribeiro-da-Silva A, Cuello AC (1990) Choline acetyltransferase-immunoreactive profiles are presynaptic to primary sensory fibers in the rat superficial dorsal horn. J Comp Neurol 295:370-384.

Ribeiro-da-Silva A, Pignatelli D, Coimbra A (1985) Synaptic architecture of glomeruli in superficial dorsal horn of rat spinal cord, as shown in serial reconstructions. J Neurocytol 14:203-220.

Ribeiro-da-Silva A, Piorio EP, Cuello C (1991) Substance P- and enkephalin-like immunoreactivities are colocalized in certain neurons of the substantia gelatinosa of the rat spinal cord: an ultrastructural double-labeling study. J Neurosci 11:1068-1080.

Robertson B, Grant G (1985) A comparison between wheat germ agglutinin and choleragenoid-horseradish peroxidase as anterogradely transported markers in central branches of primary sensory neurones in the rat with some observations in the cat. Neuroscience 14:895905.

Schmidt HHHW, Gagne GD, Nakane M, Gorsky LD, Forstermann U, Murad F (1992) Mapping of neural nitric oxide synthase in the rat suggests frequent co-localization with NADPH diaphorase but not soluble guanylyl cyclase and novel paraneural functions for nitrinergic signal transduction. J Histochem Cytochem 40:1439-1456.

Schneider SP, Perl ER (1985) Selective excitation of neurons in the mammalian spinal dorsal horn by aspartate and glutamate in vitro: correlation with location and excitatory input. Brain Res 360:339343.

Schouenborg J, Sjölund BH (1986) First-order nociceptive synapses in rat dorsal horn are blocked by an amino acid antagonist. Brain Res 379:394-398.

Skilling SR, Smullin DH, Beitz AJ, Larson AA (1988) Extracellular amino acid concentrations in the dorsal spinal cord of freely moving rats following veratridine and nociceptive stimulation. J Neurochem 51:127-132.

Sorkin LS, McAdoo DJ(1993) Amino acids and serotonin are released 
into the lumbar spinal cord of the anesthetized cat following intradermal capsaicin injections. Brain Res 607:89-98.

Spike RC, Todd AJ, Johnston HM (1993) The coexistence of NADPH diaphorase with GABA, glycine and acetylcholine in rat spinal cord. J Comp Neurol 335:320-333.

Todd AJ, Spike RC (1993) The localization of classical transmitters and neuropeptides within neurons in laminae I-III of the mammalian spinal dorsal horn. Prog Neurobiol 41:609-645.

Tracey DJ, DeBiasi S, Phend KD, Rustioni A (1991) Aspartate-like immunorcactivity in primary afferent neurons. Neuroscience 40:673686.

Valtschanoff JG, Weinberg RJ, Rustioni A (1992a) Peripheral injury and anterograde transport of wheat germ agglutinin horseradish peroxidase to the spinal cord. Neuroscience 50:685-696.

Valtschanoff JG, Weinberg RJ, Rustioni A (1992b) NADPH diaphorase in the spinal cord of rats. J Comp Neurol 321:209-222.

Valtschanoff JG, Weinberg RJ, Rustioni A, Schmidt HHHW (1992c) Nitric oxide synthase and GABA colocalize in lamina II of rat spinal cord. Neurosci Lett 148:6-10.

Valtschanoff JG, Weinberg RJ, Kharazia V, Schmidt HHHW, Nakane M, Rustioni A (1993a) Neurons in rat cerebral cortex that synthesize nitric oxide: NADPH diaphorase histochemistry, NOS immunocytochemistry, and colocalization with GABA. Neurosci Lett 157:157161.

Valtschanoff JG, Weinberg RJ, Kharazia VN, Nakane M, Schmidt HHH (1993b) Neurons in rat hippocampus that synthesize nitric oxide. $J$ Comp Neurol 331:111-121.

Valtschanoff JG, Phend KD, Bernardi PS, Weinberg RI, Rustioni A (1994) Amino acid immunocytochemistry of primary afferent terminals in the rat dorsal horn. J Comp Neurol 346:237-252.

Weinberg RJ, Van Eyck SL (1991) A tetramethylbenzidine/tungstate reaction for horseradish peroxidase histochemistry. J Histochem $\mathrm{Cy}-$ tochem 39:1143-1148.

Willis WD, Coggeshall RE (1991) Sensory mechanisms of the spinal cord, $2 \mathrm{~d}$ ed. New York: Plenum.

Womack MD, MacDermott AB, Jessel TM (1988) Sensory transmitters regulate intracellular calcium in dorsal horn neurons. Nature 334: 351-353.

Yoshimura M, Jessell TM (1990) Amino acid mediated EPSPs at primary afferent synapses with substantia gelatinosa neurones in the rat spinal cord. J Physiol (Lond) 430:315-335. 